\title{
Characterisation of the Resistance Patterns to Non Beta-Lactam Antimicrobials in Esbl-Producing Enterobacteriaceae Isolated from Dogs and Their Owners
}

\author{
Andreea Paula COZMA ${ }^{* 1}$, Iulia Elena MĂCIUCĂ², Cătălin CARP-CĂRARE ${ }^{1}$, Cristina RIMBU ${ }^{1}$, Eleonora
} GUGUIANU $^{1}$, Mihai CARP-CĂRARE ${ }^{1}$, Dorina TIMOFTE ${ }^{1,2}$

${ }^{1}$ Department of Public Health, Faculty of Veterinary Medicine, M. Sadoveanu Street, No. 8, Iaşi.

${ }^{2}$ Institute of Veterinary Sciences, University of Liverpool, Leahurst Campus Neston

Cheshire CH64 7TE, Liverpool, United Kingdom

*corresponding author: andreeapaulacozma@yahoo.com

Bulletin UASVM Veterinary Medicine 75(1)/2018

Print ISSN 1843-5270; Electronic ISSN 1843-5378

doi:10.15835/buasvmcn-vm:003917

\begin{abstract}
Enterobacteriaceae producing extended-spectrum beta-lactamase (ESBL) enzymes are resistant to betalactam agents and are also commonly multidrug resistant being associated with the resistance to other classes of antibiotics.

The aim of our study was to characterise resistance patterns in non-beta-lactam antibiotics of ESBL-producing Enterobacteriaceae strains isolated from faecal matter of pets and owners.

The study was carried out on 63 samples of faecal matter (42 from pets and 21 from owners). The ESBL screening was carried out using the Brilliance ESBL Oxoid chromogenic medium. The isolated strains that generated characteristic presumptive ESBL-producing colonies were cultivated on 5\% sheep blood medium for the extraction of bacterial DNA using the boiled preps technique. The confirmation of E. coli species was performed molecularly based on the detection of $b l a_{\text {uidA }}$ and $b l a_{\text {uspA }}$ genes. Other Enterobacteriaceae species were identified based on the minimum biochemical characteristics using the MIU and TSI medium. The phenotypical confirmation of presumptive ESBL-producing strains was carried out using the Double Disc Synergy Test (DDST) using a combination of $3^{\text {rd }}$ generation cephalosporins and beta-lactamase inhibitor agents. The determination of the resistance degree in other classes of antibiotics was carried out through the Kirby-Bauer diffusimetric method, and the results were interpreted according to the CLSI standard.

Following the species investigation of isolates, 60/63 (95.28\%) belonged to the E. coli species and 3/63 $(4.72 \%)$ to the $K$. pneumoniae species. Animal isolates were resistant to sulphonamides $(54.76 \%$ resistance to SXT), fluoroquinolones ( $45.23 \%$ resistance to ENR) and tetracyclines (54.75\% resistance to TE). In addition to strains of animal origin for isolates of human origin, an increased resistance has been noticed to phenicols and aminoglycosides.

This study has identified a high prevalence of ESBL-producing Enterobacteriaceae strains and associated with multidrug resistance for pets and their owners.
\end{abstract}

Keywords: dogs, owners, ESBL, Enterobacteriaceae, antibioresistance

\section{Introduction}

The emergence of infections with extendedspectrum beta-lactamase enzyme-producing bacteria is continuously rising, and the resistance mechanism determined by these enzymes influen- ces the first-line treatment with beta-lactams in anti-infectious therapy both of human and of animal origin (Nóbrega, 2014). The resistance determined by the ESBL enzymes is commonly associated with resistance to other antimicrobial 
classes, the phenomenon of in-cross resistance and multidrug resistance being often reported in extended-spectrum beta-lactamase enzymeproducing Enterobacteriaceae strains (Ewers, 2012). In Romania, the studies carried out for the characterisation of resistance associated to ESBLproducing Enterobacteriaceae strains are rare and carried out particularly on strains of human origin.

The aim of the study was to characterise the resistance phenotype in non-beta-lactam antibiotics of ESBL-producing Enterobacteriaceae strains isolated from faecal matter of pets and owners.

\section{Materials and methods}

The study was carried out on 63 ESBLproducing Enterobacteriaceae strains (42 of animal origin and 21 of human origin) and isolated from faecal matter samples collected from animals with the sterile swab from the rectum, while the owners used stool culture containers.

After collection, the faecal matter samples were impregnated on Oxoid Brilliance ESBL Agar medium (Oxoid, Basingstoke, UK), a medium specific for isolating ESBL-producing Enterobacteriaceae (Huang, 2010). The isolated strains that produced characteristic colonies on the medium used for screening were considered presumptive ESBL and cultivated on blood agar medium (Blood Agar Oxoid) for the extraction of bacterial DNA using the boiled preps technique in order to achieve taxonomic classification of isolated strains.

The E.colistrains were taxonomically classified through the PCR identifying the bla $a_{\text {uidA }}$ and $b l a_{\text {uspa }}$ genes keeping the protocol recommended by Anastasi et al. (2010), and McDaniels et al. (1996), respectively. The other Enterobacteriaceae were taxonomically classified based on some minimum biochemical characteristics using the MIU and TSI media. The phenotypical confirmation of presumptive EBSL-producing strains was carried out through the combination disc test, a method recommended by the CLSI standard (CLSI, 2014).

The characterisation of the resistance phenotype in other non-beta-lactam antibiotics of analysed strains was carried out through the diffusimetric method (Kirby Bauer) using antibiotics from the following classes: fluoroquinolones, aminoglycosides, phenicols, sulphonamides, tetracyclines. The chosen antibiotics were selected according to the principle of classification of the isolated strains in the MDR (multidrug resistance) category (Magiorakos, 2012). The interpretation of the results was carried out according to the CLSI standard (2).

\section{Results and discussion}

Following processing of faecal matter samples, 63 presumptive EBSL-producing Enterobacteriaceae strains have been isolated (42 of animal origin and 21 of human origin).

In regard to the spectrum of isolated strains, of the 63 Enterobacteriaceae strains, 60 (95.28\%) belonged to the E. coli species and $3(4.72 \%)$ to the $K$. pneumoniae species. All Enterobacteriaceae strains isolated following ESBL screening were phenotypically confirmed through the combination disc test as being extended-spectrum betalactamase enzyme-producing Enterobacteriaceae.

The investigations carried out to characterise the resistance degree to other classes of nonbeta-lactam antibiotics revealed, for the strains of animal origin, a high degree of resistance for the antibiotics from the following classes: sulphonamides $(23 / 42 ; 54.76 \%$ resistance to sulfamethoxazole/trimethoprim), fluoroquinolones (19/42; $45.23 \%$ resistance to enrofloxacin), and tetracyclines $(23 / 42 ; 54.76 \%$ resistance to tetracycline) (figure 1). The isolated strains of human origin were characterised by a high degree of resistance to the antibiotics from the following classes: tetracyclines $(15 / 21 ; 71.43 \%$ resistance to tetracycline), sulphonamides $(12 / 21 ; 57.14 \%$ resistance to sulfamethoxazole/trimethoprim), phenicols $(10 / 21 ; 47.62 \%$ resistance to chloramphenicol) and aminoglycosides $(10 / 21 ; 47.62 \%$ resistance to gentamicin) (figure 2).

The results obtained following the conducted investigations are in accordance with the specialised literature. Moreover, they are supported, if we associate the results with the statistics regarding the consumption of antibiotics in Romania, both in human medicine, and in veterinary medicine. In 2015, the reports of the ECDC (European Center for Diseases Prevention and Control) and CDDEP (Center for Disease Dynamics, Economics and Policy) presented a high consumption of antibiotics from the classes: beta-lactams, quinolones, sulphonamides and aminoglycosides (Gelband, 2015; ECDC, 2015). In regard to the consumption of antibiotics in veterinary medicine, according to the report of 


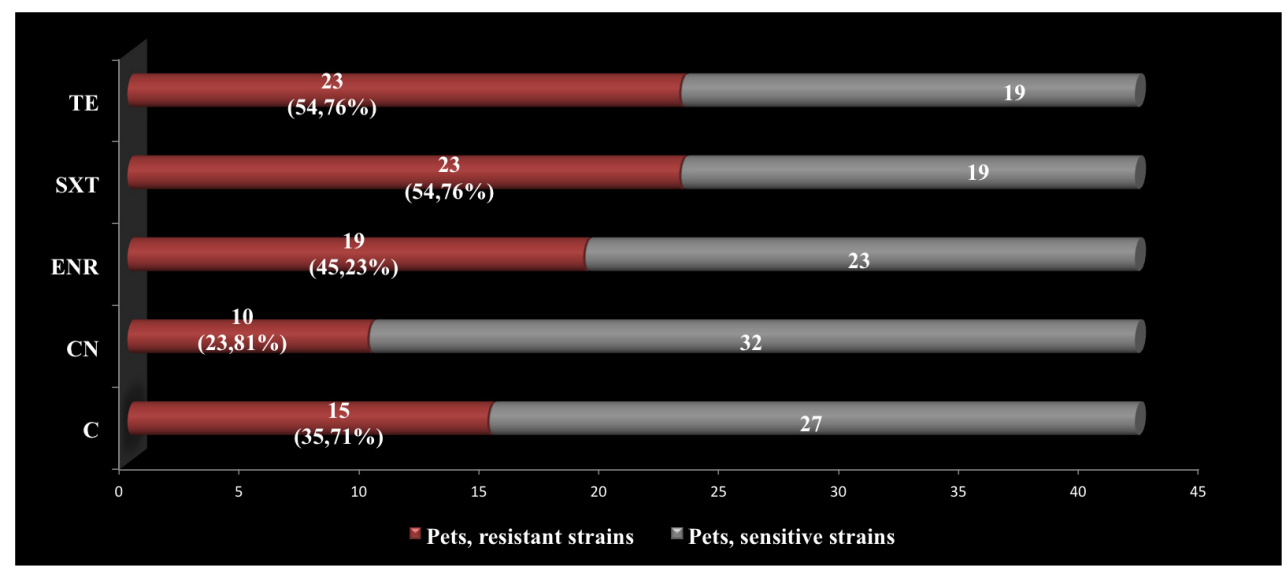

Figure 1. Resistance of ESBL-producing bacterial strains to other classes of antibiotics

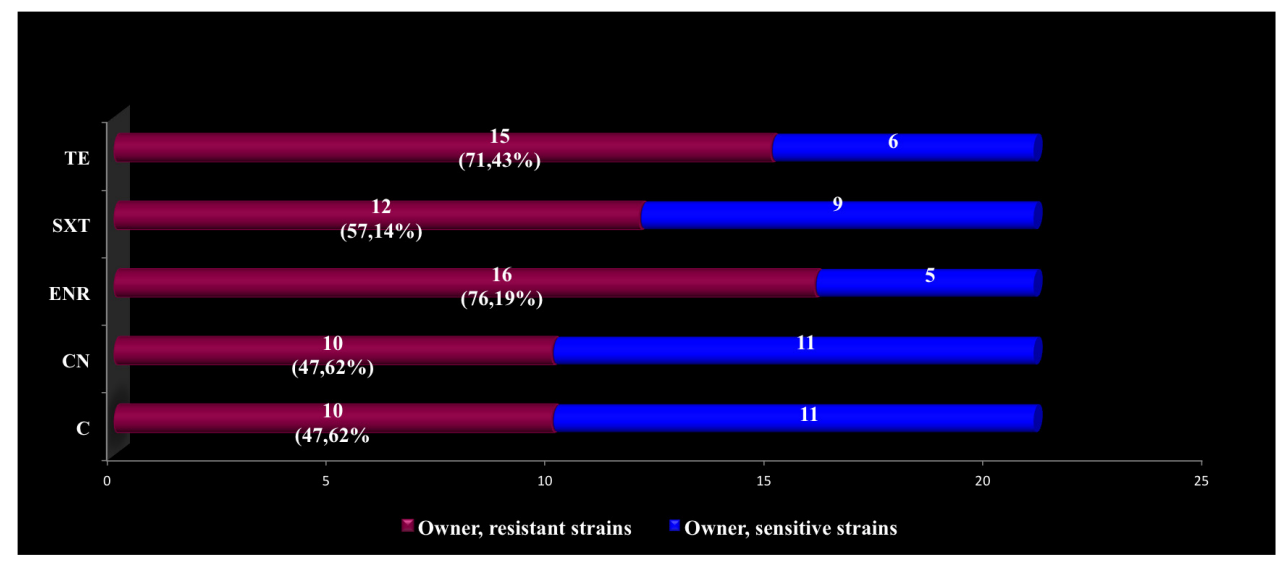

Figure 2. Resistance of ESBL-producing bacterial strains, isolated from owners, to other classes of antibiotics

the European Medicines Agency from 2016, the antibiotics from the classes: tetracyclines, betalactams, sulphonamides and aminoglycosides are the most used in the treatment of infectious diseases of animal origin (European Medicine Agency, 2016).

A bacterial strain resistant to antibiotics from at least 3 different classes of antibiotics is associated with multidrug resistance and classified in the MDR category (8). According to the previously presented results, this study has identified a high prevalence of ESBL-producing bacterial strains and associated with multidrug resistance. Thus, from the total of 42 strains of animal origin, $39(92.85 \%)$ have been classified in the MDR category. For the isolated strains of human origin, 19/21 (90.47\%) were MDR.

\section{Conclusion}

This study has identified a high prevalence of ESBL-producing Enterobacteriaceae strains and associated with multidrug resistance for pets and their owners.

Acknowledgments. This research did not receive any specific grant from funding agencies in the public, commercial, or not-for-profit sectors.

\section{References}

1. Anastasi EM, Matthews B, Gundogdu A, Vollmerhausen TL, Ramos NL, Stratton H, Ahmed W, Katouli1 M (2010). Prevalence and Persistence of Escherichia coli Strains with Uropathogenic Virulence Characteristics in Sewage Treatment Plants. Applied and Environmental Microbiology, 76: 5882-5886.

2. Clinical and Laboratory Standards Institute (2014). Performance Standards for antimicrobial susceptibility testing: Twenty-fourth International Supplement M100-S24, Wayne, PA, USA: CLSI.

3. European Centre for Disease Prevention and Control (2015). Sinteza ultimelor date privind consumul de antibiotice în UE, pp.1-11.

4. European Medicines Agency (2016). Sales of veterinary antimicrobial agents in 29 European countries in 2014, Trends from 2011 to 2014, EMA/61769/2016. 
5. Ewers C, Bethe A, Semmler T, Guenther S, Wieler LH (2012). Extended-spectrum blactamase-producing and AmpC-producing Escherichia coli from livestock and companion animals, and their putative impact on public health: a global perspective. Clinical Microbiology and Infection, 18: 646-655.

6. Gelband H, Miller-Petrie M, Pant S, Gandra S, Levinson J, Barter D, White A, Laxminarayan R (2015). The State of the world's antibiotics 2015. Center for Disease Dynamics, Economics \& Policy, 1-84.

7. Huang T-D, Bogaerts P, Berhin C, Guisset A, Glupczynski Y (2010). Evaluation of Brilliance ESBL Agar, a Novel ChromogenicMedium forDetection ofExtended-SpectrumBeta-Lactamase-Producing Enterobacteriaceae. Journal of Clinical Microbiology, 48: 2091-2096.

8. Magiorakos AP, Srinivasan A, Carey RB, Carmeli Y, Falagas ME, Giske CG, Harbarth S, Hindler JF, Kahlmeter G, OlssonLiljequist B, Paterson DL, Rice LB, Stelling J, Struelens MJ,
Vatopoulos A, Weber JT, Monnet DL (2012). Multidrugresistant, extensively drug-resistant and pandrugresistant bacteria: an international expert proposal for interim standard definitions for acquired resistance. European Society of Clinical Microbiology and Infectious Diseases, 18: 268-281.

9. Mcdaniels AE, Rice EW, Reyes AL, Johnson CH, Haugland RA, Stelma GN Jr (1996). Confirmational Identification of Escherichia coli, a Comparison of Genotypic and Phenotypic Assays for Glutamate Decarboxylase and b-DGlucuronidase. Applied and Environmental Microbiology, 62: 3350-3354.

10. Nóbrega DB, Brocchi M (2014). An overview of extendedspectrum beta-lactamases in veterinary medicine and their public health consequences. Journal of Infection in Developing Countries, 8: 954-960. 